\title{
The use of experimental vignette studies to identify drivers of variations in the delivery of health care: a scoping review
}

\author{
Jessica Sheringham ${ }^{1 *}$, Isla Kuhn² and Jenni Burt ${ }^{2}$
}

\begin{abstract}
Background: Identifying how unwarranted variations in healthcare delivery arise is challenging. Experimental vignette studies can help, by isolating and manipulating potential drivers of differences in care. There is a lack of methodological and practical guidance on how to design and conduct these studies robustly. The aim of this study was to locate, methodologically assess, and synthesise the contribution of experimental vignette studies to the identification of drivers of unwarranted variations in healthcare delivery.

Methods: We used a scoping review approach. We searched MEDLINE, Embase, Web of Science and CINAHL databases (2007-2019) using terms relating to vignettes and variations in healthcare. We screened title/abstracts and full text to identify studies using experimental vignettes to examine drivers of variations in healthcare delivery. Included papers were assessed against a methodological framework synthesised from vignette study design recommendations within and beyond healthcare.
\end{abstract}

Results: We located 21 eligible studies. Study participants were almost exclusively clinicians (18/21). Vignettes were delivered via text $(n=6)$, pictures $(n=6)$, video $(n=6)$ or interactively, using face-to-face, telephone or online simulated consultations $(n=3)$. Few studies evaluated the credibility of vignettes, and many had flaws in their wider study design. Ten were of good methodological quality. Studies contributed to understanding variations in care, most commonly by testing hypotheses that could not be examined directly using real patients.

Conclusions: Experimental vignette studies can be an important methodological tool for identifying how unwarranted variations in care can arise. Flaws in study design or conduct can limit their credibility or produce biased results. Their full potential has yet to be realised.

Keywords: Vignettes, Healthcare variation, Inequalities

\section{Introduction}

Unwarranted variations in the delivery of health care are widespread [1,2]. These variations have manifested in systematically poorer quality or lower availability of care for patients for reasons including their gender, age, ethnicity, and socioeconomic circumstances [3]. Examples

\footnotetext{
* Correspondence: j.sheringham@ucl.ac.uk

'Department of Applied Health Research, UCL, 1-19 Torrington Place, London WC1E 7HB, UK

Full list of author information is available at the end of the article
}

of such inequalities include patients of Hispanic or South Asian ethnic backgrounds reporting poorer experience of their doctors than majority white patients in the USA and UK [4-7], and delays in cancer diagnosis (associated with poorer survival) being reported more frequently for older patients and patients in adverse socioeconomic circumstances compared to younger and majority white patients in the UK $[8,9]$. Evidence on how such variations arise and persist is required to inform improvement efforts. Proposed drivers of variations

C C The Author(s). 2021 Open Access This article is licensed under a Creative Commons Attribution 4.0 International License, which permits use, sharing, adaptation, distribution and reproduction in any medium or format, as long as you give appropriate credit to the original author(s) and the source, provide a link to the Creative Commons licence, and indicate if changes were made. The images or other third party material in this article are included in the article's Creative Commons licence, unless indicated otherwise in a credit line to the material. If material is not included in the article's Creative Commons licence and your intended use is not permitted by statutory regulation or exceeds the permitted use, you will need to obtain permission directly from the copyright holder. To view a copy of this licence, visit http://creativecommons.org/licenses/by/4.0/ The Creative Commons Public Domain Dedication waiver (http://creativecommons.org/publicdomain/zero/1.0/) applies to the data made available in this article, unless otherwise stated in a credit line to the data. 
in the delivery of care include individual healthcare provider perceptions or behaviours - such as the presence of implicit bias [10] - as well as variations in patient expectations or behaviours [11]. Differences in how decisions are reached as providers and patients interact may also contribute to persistent variations in care [12]. These explanations are widely proposed in many areas where variations are identified, but robust evidence often remains lacking or inconclusive [13, 14]. Obtaining actionable insights into the judgements, activities and behaviours of individuals within health care systems is challenging. It is even more challenging when the situations under scrutiny are rare, occur in complex settings, or raise difficult ethical questions [15]. Experimental vignette studies offer one methodological approach to tackling this challenge.

A vignette is a short, carefully constructed depiction of a person, object, or situation, representing a systematic combination of characteristics [16]. First used in ethnographic fieldwork to prompt informants for more detailed reflection [17], hypothetical scenarios were subsequently adopted by experimental psychologists to examine cognitive processes $[18,19]$. Vignette approaches have since been taken up in diverse fields including social science $[15,20,21]$, organisational research [22, 23], applied and social psychology [24], business ethics [25], information studies [26], and nursing research [27].

In experimental vignette studies, vignettes are used to explore participants' attitudes, judgements, beliefs, emotions, knowledge or likely behaviours by presenting a series of hypothetical yet realistic scenarios across which key variables have been intentionally modified whilst the remaining content of the vignette is kept constant [22, 26]. Such studies seek to generate inferences about cause-and-effect relationships by considering the nature of each vignette, and participants' subsequent responses to these vignettes $[28,29]$. Vignettes themselves may be presented using a variety of modalities, including text, pictures, video or by using actors in simulated or real clinical environments. Studies are often factorial in design, with vignettes created to represent all possible combinations of pre-defined factors of interest, and a random sample of vignettes subsequently presented to each participant $[18,27,30]$. Experimental vignette studies provide a 'hybrid' approach between conventional surveys and observations of real-life practice. The intentional manipulation of vignettes in experimental designs to compare the causal effects of variables enhances internal validity, whilst the survey sampling approaches available to researchers conducting vignettebased studies enhances external validity $[16,22,31]$.

Opponents to vignette studies commonly note that they are not studying real life $[26,32]$. Several validation studies have examined how vignettes perform against alternative methods of assessing the delivery of care, often using medical records and standardised patients as comparators [33-35]. Whilst each method inevitably has strengths and weaknesses, well designed vignette studies may have advantages in certain scenarios. Health care professionals' choices of care in clinical vignettes have been found to reflect their stated intentions and behaviours more closely than data extracted from medical records or from recordings of real consultations [33-35]. Biases or inaccuracies may arise from observations of actual clinical practice in a number of ways. For example, evidence suggests that physicians may under-report clinical activities within medical records, possibly due to time constraints [33]. Additionally, key actions can be missed in recording doctor-patient consultations; body language is omitted from analyses of audio recordings, whilst off-camera events are missed in video recordings $[36,37]$. As a result, observational studies alone may not provide sufficient depth of evidence to inform successful efforts to reduce variations in care; experimental vignette studies offer an alternative lens through which to identify key drivers of variations.

In our experience of conducting experimental vignette studies, there is a lack of methodological and practical guidance available on how to design and conduct these studies robustly. Unlike other study types, there is no universal checklist to ensure vignette studies are understandable, transparent and of high quality [38]. The aim of this scoping review was to locate, methodologically evaluate, and synthesise the contribution of experimental vignette studies that seek to identify drivers of unwarranted variations in the delivery of healthcare. In doing so, we hope to provide an overview of how to do such studies well, and what we can learn from them.

\section{Methods}

We conducted a scoping review in accordance with PRISMA-ScR guidelines [39].

\section{Eligibility criteria}

We aimed to locate primary empirical studies that used an experimental vignette design to examine drivers of variation in the delivery of healthcare. The review focused on drivers of variation and therefore excluded those that only sought to describe variations, as the measurement of variations is feasible using records or observation of real healthcare delivery See supplementary file 1 for full inclusion and exclusion criteria.

\section{Information sources and search strategy}

The search strategy was developed in collaboration with an experienced information specialist (IK), and used text words and synonyms for vignettes and variations in 
healthcare (supplementary file 1). The following databases were searched from January 2007 to April 2019: MEDLINE (via Ovid), Embase (via Ovid), Web of Science, and CINAHL (via EBSCO). The search was limited to 2007 because the majority of methodological reviews of vignettes were published since this date (see supplementary file 2). The search strategy was developed in MEDLINE and adapted for other databases as appropriate.

\section{Study selection}

We used a phased approach to title/abstract screening. First, an automated search of key words in titles and abstracts was undertaken using Stata15 [40] to exclude studies that were clearly of no relevance (e.g. studies published in planetary journals). We undertook manual checks of automated Stata screening exclusions to refine terms (for example, initially terms connected with education were used to exclude studies on students but removed when they were identified as excluding papers referring to qualified physicians). Next, JS manually screened the remaining titles and abstracts to exclude papers that did not examine healthcare variations using vignettes, or that measured rather than sought to identify drivers of variation. JB double screened $10 \%$ of the full sample to confirm accuracy and clarify inclusion criteria. Inter-rater agreement was assessed using Cohen's kappa for a subset of papers. Prior to consensus discussions, Kappa was $66 \%$, which can be interpreted as moderate agreement [41].

Full-text screening was conducted by JS with $10 \%$ double screening by JB. For both title/abstract and fulltext screening, all differences were resolved by discussion.

\section{Data extraction}

Data were extracted by JS, with $10 \%$ double extraction by JB, using a tool developed and piloted for the purposes of this study, covering setting, respondents, healthcare setting, medical condition under scrutiny, patient characteristics, drivers of variation under scrutiny, and vignette modality.

\section{Methodological assessment}

There is no existing standardised approach to evaluating the robustness of experimental vignette studies. We therefore conducted a review of methodological reviews of vignette studies within and beyond healthcare. Synthesising insights from all included methodological papers $[26,32,42-46]$, we developed a framework to appraise the design and conduct of experimental vignette studies within this review: see supplementary file 2 for full details of this review of reviews and the framework development.
Within this framework, we identified factors considered important in maximising internal and external validity of experimental vignette studies in two broad areas: (A) the design and description of vignettes used, and (B) the wider study design and methods within which vignettes are employed, as outlined below (Table 1) [46, $50,52]$.

\section{A. Vignette design}

Six key considerations were identified as important in the construction and use of robust vignettes: vignette credibility, number, variability, mode, evaluation, and description. These are described in more detail in Table 1. We use the term vignette to refer to the overall description or depiction of each situation as presented to the participants. Within each vignette, experimental factor/s represent the variable/s of interest which have been intentionally modified and manipulated (such as gender or ethnicity); the representation of experimental factor/s refers to the varying ways in which each experimental factor is represented across the vignettes (e.g. the multiple ways in which ethnicity or gender have been presented to the participant).

\section{B. Wider study design}

Four considerations were identified as important in the overall design of experimental vignette studies: concealment, realism, sampling and response rates, and analysis (Table 1). In almost all cases, experimental vignette studies are a form of survey, and thus principles of good survey design (including standards for good questionnaire design) should be followed.

We applied this framework to all included studies to appraise the way in which they were conducted. We generated a scoring system to reflect how well studies had met eight of the ten methodological considerations. For four considerations (vignette credibility, evaluation, description and study analysis) the scores primarily reflected the extent to which sufficient methodological detail was provided. For two considerations (vignette variability and study realism) the score primarily reflected whether optimal choice in the design of the study was made. For two considerations in the wider study design (concealment and sampling/response), the scores reflected both provision of methodological detail and the quality of study execution. The sampling/response consideration was weighted most heavily in the scoring system (maximum of 6 marks) because we judged it of key importance to the credibility and validity of studies seeking to report on inequalities. Two considerations were not given a score; mode of vignette delivery and whether multiple vignettes were provided. Both 
Table 1 Methodological framework for assessment of experimental vignette studies
A. Vignette design
1. Credibility
- The degree to which vignettes credibly represent critical aspects of a clinical scenario or patient to potential participants is crucial to the success of an experimental vignette study [37].
- Lens model approaches (studies which compare optimal versus actual decisions in a given situation, originally developed by Brunswick in 1950) have demonstrated empirically that the decision-making performance of participants is improved when situations are realistic [47].
- Basing vignettes on real-life data, clinical expertise, and existing guidelines are recommended ways of enhancing credibility $[26,45,46]$.

2. Number

- Presenting participants with more than one vignette enables examination of variations in judgement within individuals as well as between them - that is, the extent to which each participant is differentially influenced by each experimental factor in making their decisions. Where this is required to address study aims, for example, in vignette approaches based on the lens model [48], it is typically recommended that there are at least five different representations for each experimental factor.

- Additional considerations are needed when several vignettes are used "such as controlling for the order in which vignettes are presented and taking account of clustering within individuals in the analysis (see: wider study design).

3. Variability

- Developing or using a number of different representations of each experimental factor may increase study generalisability, by reducing the possibility that idiosyncrasies in one particular representation are responsible for findings. For example, using one female and one male actor in video vignettes may lead not to participants responding to the constructs of gender, but to that particular female or that particular male.

- Where participants do view more than one vignette, analysis must account for clustering of vignettes by respondent, to avoid over-estimating the statistical significance of any effect [49].

4. Mode

- The mode through which vignettes are delivered has an important influence on the research question an experimental vignette study can answer.

- Vignette mode has historically been textual only, with participants presented with a written scenario. Text-based vignettes may constrain not just the information the respondent is given, but how this information is framed.

- More recently the use of pictures, videos, actors, and interactive environments have been developed [22, 46].

- Pictorial modes are particularly suited to examination of characteristics, such as ethnicity, where visual representation removes the need for explicit statement (and prior framing) of the characteristic.

- Studies using video vignettes extend this still further by enabling participants to form judgements on body language and speech patterns in addition to visual cues.

- Interactive formats, such as unannounced standardised patients or virtual reality set-ups, have the potential to mimic real delivery which enables exploration of how inequalities may unfold during a clinical encounter, through enabling explorations of variations in the information that clinical participants elicit from patients or in both parties' non-verbal communication. Such approaches are more complex to construct and more costly to develop than static vignette formats, which may limit their feasibility.

5. Evaluation - Evaluation of vignettes' face validity - during vignette construction and once data are collected - is key to understanding the validity of findings in studies using vignettes.

- Thinking through in advance what is needed to make particular vignettes 'successful' for their target audience will guide the nature of and approach to evaluation.

- Options include assessment by an expert panel, feedback from participants, or comparing responses to the vignettes to an additional data source such as clinical data $[26,46]$.

6. Description - Readers of vignette study papers need to be able to form their own judgments of vignette credibility. An entire vignette should be provided to enable them to do so.

B. Wider study design

1. Concealment - When investigating unwarranted variations in care, it is important to conceal the purpose of such studies, given that few people will volunteer behaviours or attitudes that they recognise as poor or biased.

- If the study's purpose is not adequately masked it can bias results, even with carefully constructed vignettes [31]. Participants may learn of the study purpose directly (from study information shared at recruitment) but also may infer it indirectly, through other cues in study materials (e.g. funder's name), or pre-specified responses that prime participants to consider certain answers.

2. Realism - External validity of vignette studies is enhanced when studies are conducted in a setting as close as possible to the natural ecology of decision-making $[47,50]$.

- The generalisability of studies to investigate unwarranted variation in healthcare may be improved by collecting data in a setting that mimics key aspects of clinical settings, whether that be the actual environment, other inclusion of features such as the imposition of time constraints.

3. Sampling \& response
- The representativeness of any survey rests on sampling, coverage, and nonresponse.

- This is particularly important for studies of healthcare variations, where a biased sample or responses - for physician or patient participants - may lead to over- or under-estimation of variations.

- Studies need to justify their sample design, sample size, approach to recruitment, response and completion rates, and reasons for excluding data [51].

- The implications of low or biased responses should be considered.

4. Analysis - Experimental vignette studies are often complex in how data are structured. Analysis must appropriately account for hierarchies within the data [22]. 
these considerations - while important for researchers to consider when designing vignettes - are not, intrinsically, markers of quality.

Adding up assessments across each methodological consideration, studies were then assigned to one of three groups: good, moderate or low overall methodological quality (see Table 3 and supplementary file 2 for full details of categorisation). The cut-offs were agreed by JB and JS in part determined by their overall score and in part determined by their performance on key considerations. Studies were considered moderate rather than high quality when overall their design and reporting was good enough overall but where there were significant flaws in at least one dimension. The distinction between moderate and low quality was made where we judged studies to be too flawed to inform wider understanding of healthcare inequalities.

\section{Data synthesis}

Studies were synthesised narratively, paying particular attention to how studies yielded insights into variations in healthcare delivery. We excluded studies judged to be of low methodological quality from this synthesis.

\section{Registration}

As a methodological scoping review, the study was not eligible to be registered on PROSPERO.

\section{Results}

\section{Study selection and characteristics}

We identified 23 papers and 21 unique studies for inclusion within the review (see PRISMA flowchart, Figure 1). Most studies related to primary care settings (see Table 2 for details). Studies were most frequently based in the USA $(n=14)$, with England $(n=2)$, Portugal $(n=1)$, Sweden $(\mathrm{n}=1)$, the Netherlands (1), France (1) and multi-country settings $(n=3)$ also represented. Vignette participants were almost exclusively healthcare providers $(20 / 23)$, predominantly doctors $(n=14)$. Only three studies examined public perspectives on healthcare delivery $[58,62,74]$.

Most studies (17/21) sought to examine drivers of variations in healthcare in relation to patient ethnicity. Drivers of variation were also examined in relation to patient gender $(n=9)$, socioeconomic circumstances $(n=7)$ and age $(n=9)$. No studies examined unwarranted variations by other characteristics protected in legislation in some countries, such as disability and sexuality.

\section{Methodological assessment}

We assessed ten studies as being of good methodological quality (Table 3). We focused on these studies in exploring how vignettes may produce insights into drivers of variations of care. Seven studies were assessed as moderate quality, with lower certainty about the insights they could provide into drivers of healthcare variation. Four studies were assessed as low methodological quality, primarily because flaws in their sampling and response rates led to the possibility of significant biases that would compromise the validity of their findings, no matter how well their vignettes were designed and executed. More details on how the 21 included studies were designed and conducted are given below.

\section{Vignette design \\ Credibility}

Most studies provided comprehensive descriptions of how vignettes were constructed. Higher quality studies described how input from clinicians or patients influenced content and delivery. For example, Burt et al. based vignettes on previously video-recorded patientclinician encounters [42]. In a three studies, content was based on national guidelines $[65,72,75]$.

\section{Number}

Just over half $(12 / 21)$ of studies showed participants more than one vignette.

\section{Variability}

Eight high and one moderate quality study used variants of experimental factors, depicting the same experimental characteristic using more than one actor, photo or video or simulated case.

\section{Modality}

In six studies, vignette information was purely textual; here, manipulated characteristics and their variations were therefore stated clearly to participants. In 12 studies, vignette information was visual, either pictorial $(\mathrm{n}=$ 6) or video-based $(n=6)$. Here, manipulated characteristics were communicated non-verbally and may (or may not) have been inferred by the participants. In three studies, vignettes were presented interactively, with one study each using online, telephone and in-person standardised patient approaches. In interactive modalities the content of the vignette could vary across participants, as the vignette evolved in response to respondent behaviours, such as the questions they asked [42].

\section{Evaluation}

Three high quality studies comprehensively reported how their vignettes performed, most commonly in tests of credibility [58, 60, 68]. Mckinlay et al., Hirsh and Lutfey et al. used post-study quantitative surveys of participants to find out whether vignette 'patients' were typical of the real patients they encountered $[64,66,68]$. McKinlay reported that $91 \%$ of participants viewed the vignettes as typical of 
23,393 records identified through database searching

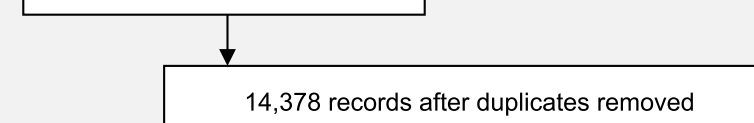

14,378 records after duplicates removed

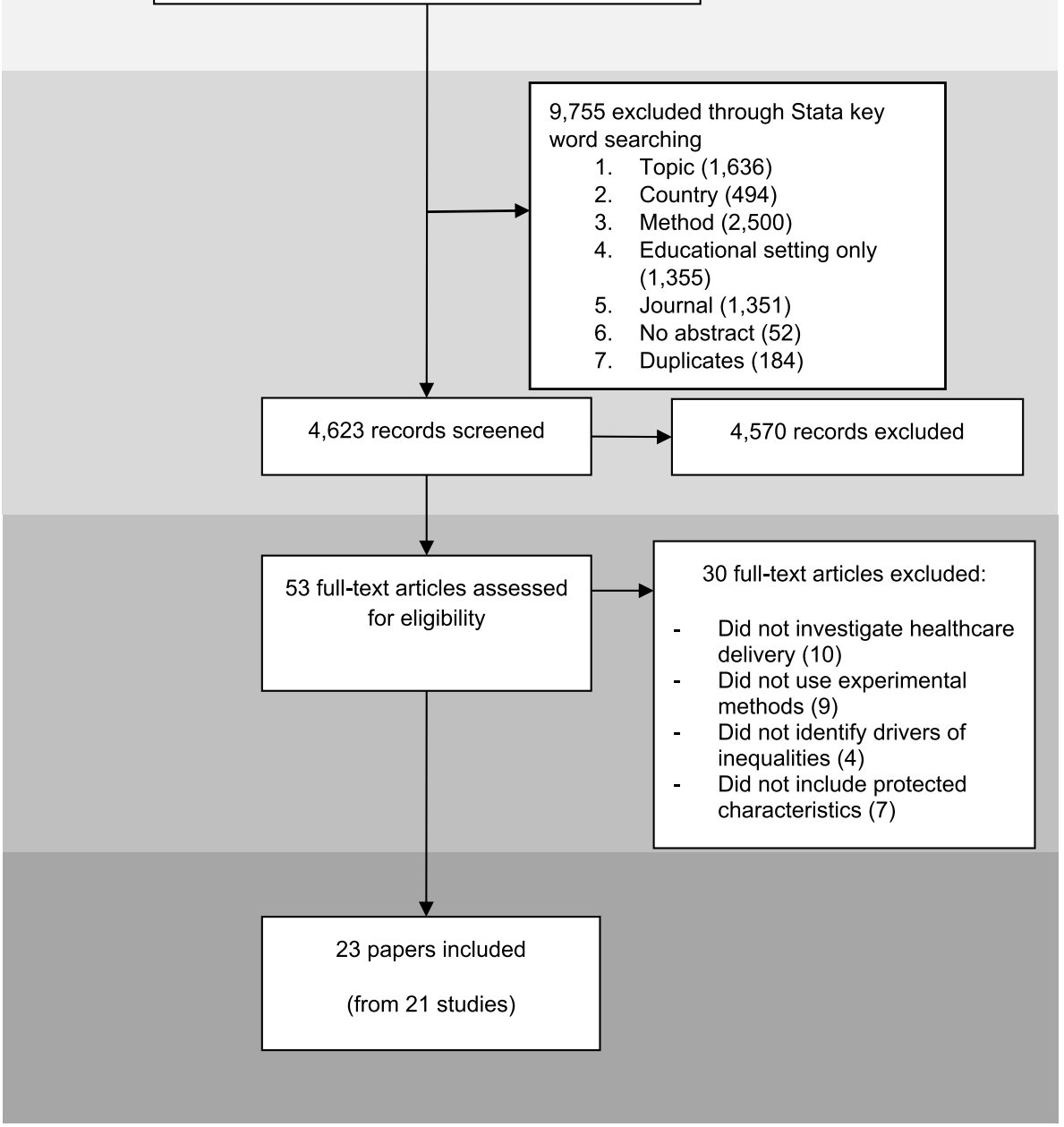

Fig. 1 PRISMA Flow diagram

their patients [68]. Burt et al reported the expert clinical raters' scores of their high and low performing vignette consultations as an indication of their credibility [58].

Vignettes performance was evaluated in other ways too. For example, Sheringham et al. had developed an online interactive vignettes application specifically for the study [72]. The authors quantified system errors that occurred when the software could not answer a question entered by a participant. System errors occurred on average in just under $5 \%$ of all participant interactions. Analysis was adjusted to examine whether system errors could have been responsible for the findings and this was found not to be the case [72]. Description of any kind of vignette evaluation were largely absent from lower quality studies.

\section{Description}

Thirteen out of 21 studies presented or facilitated access to an entire example vignette. Access to video or interactive vignettes in a journal article is not straightforward, but five out of the nine video or interactive papers did include sufficient aspects (e.g. using video stills [60]) or online links (e.g. to a multimedia demonstration [72]) to enable readers to judge vignettes' quality and credibility. 


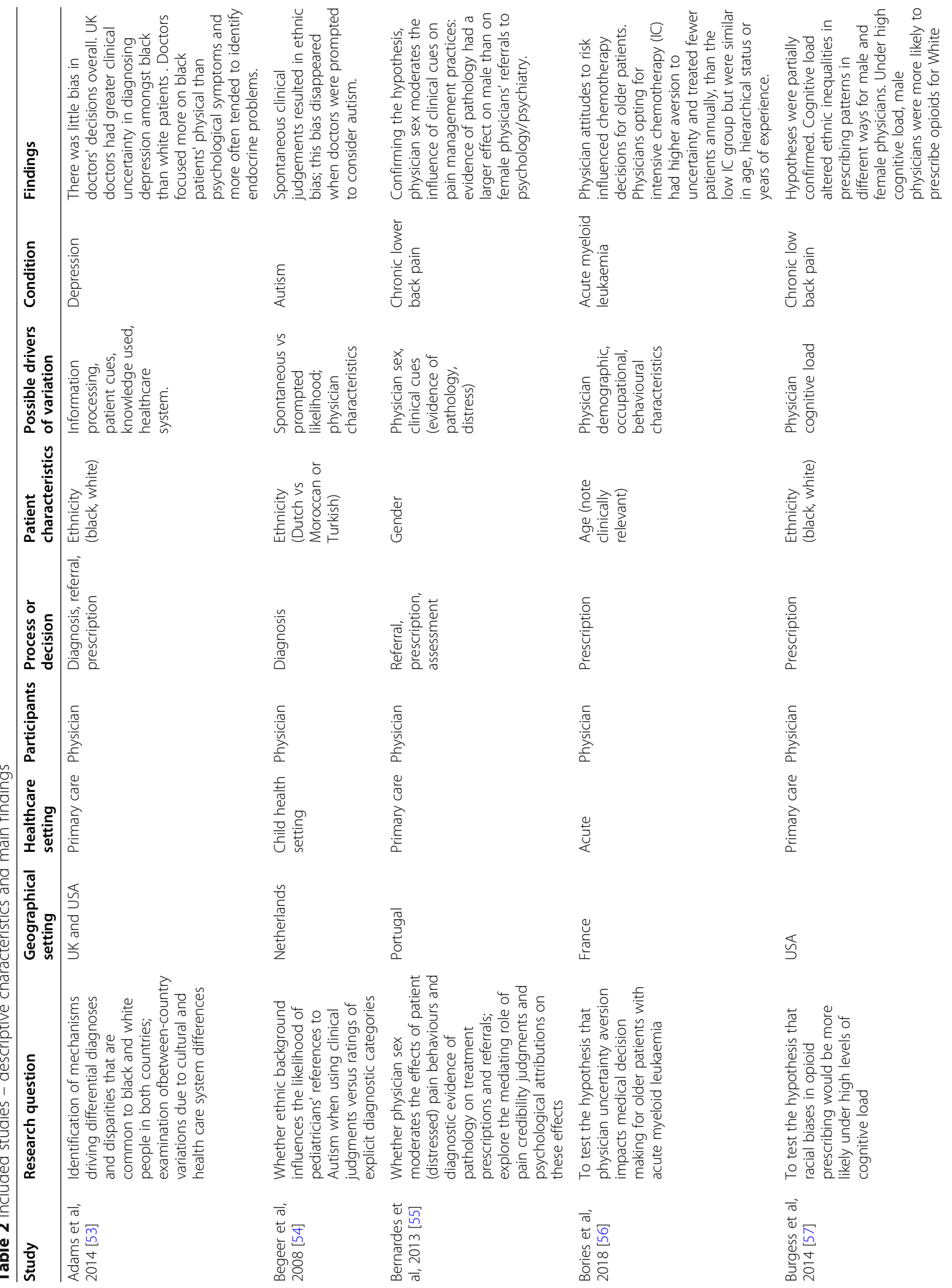




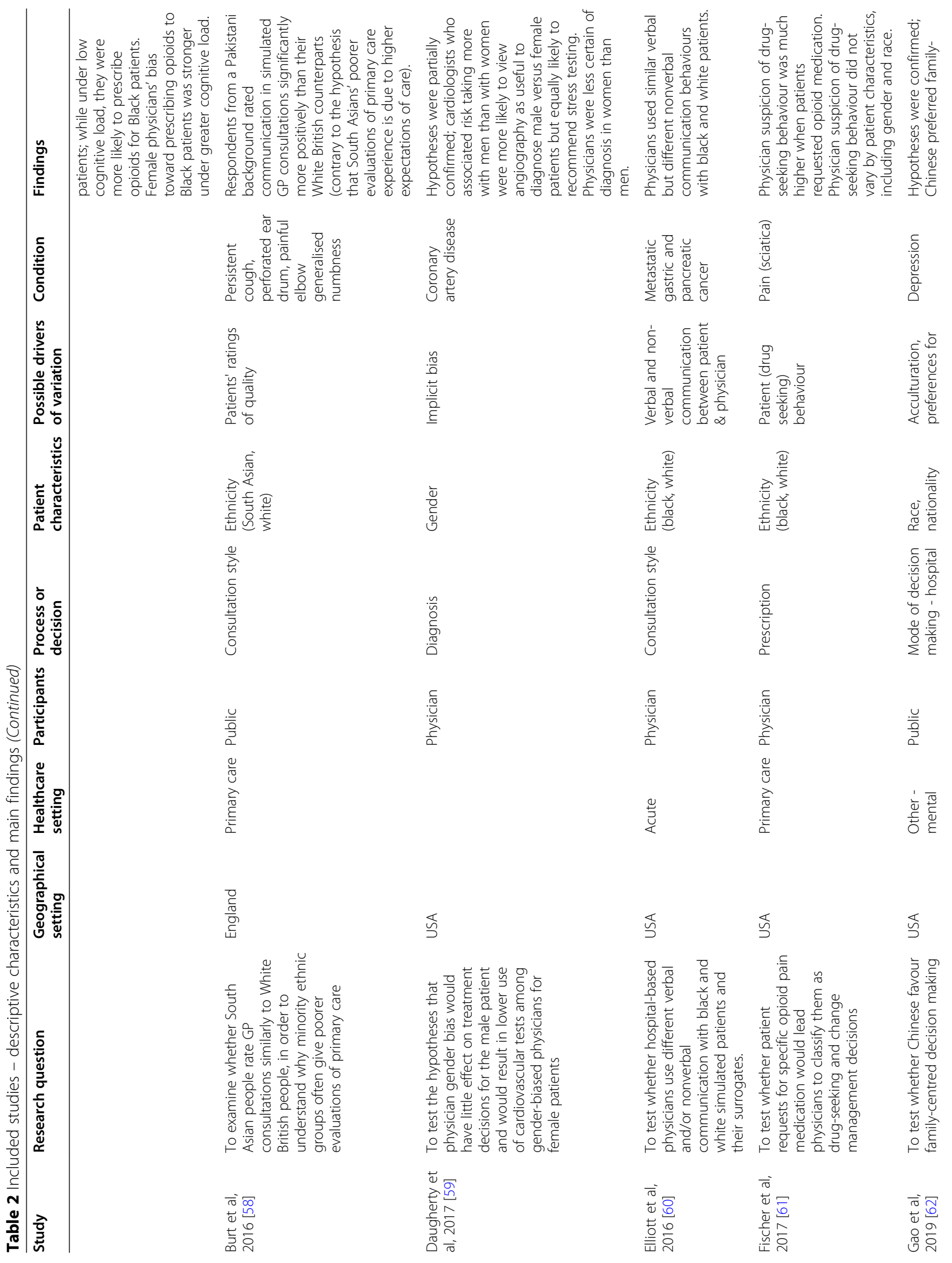




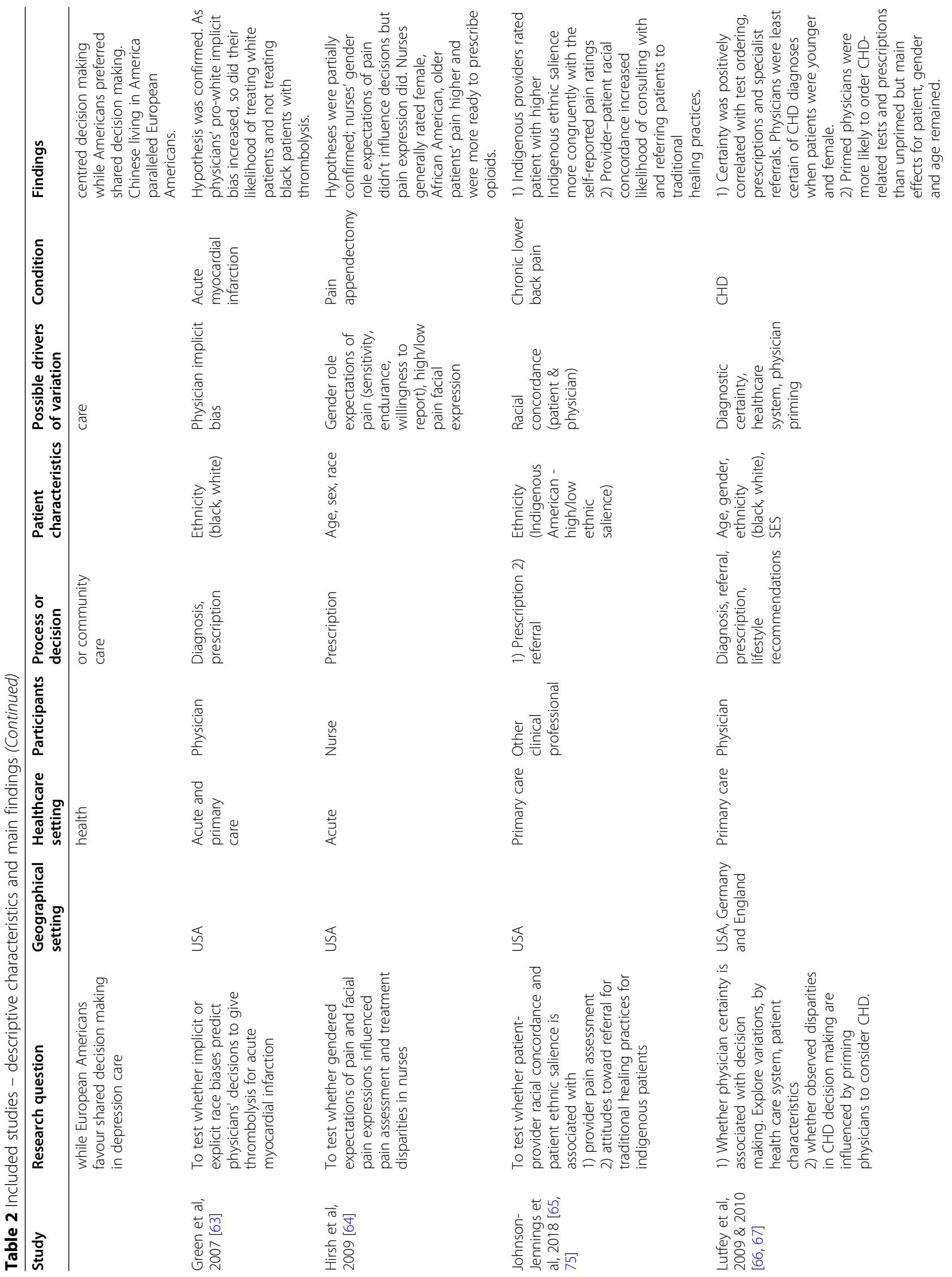




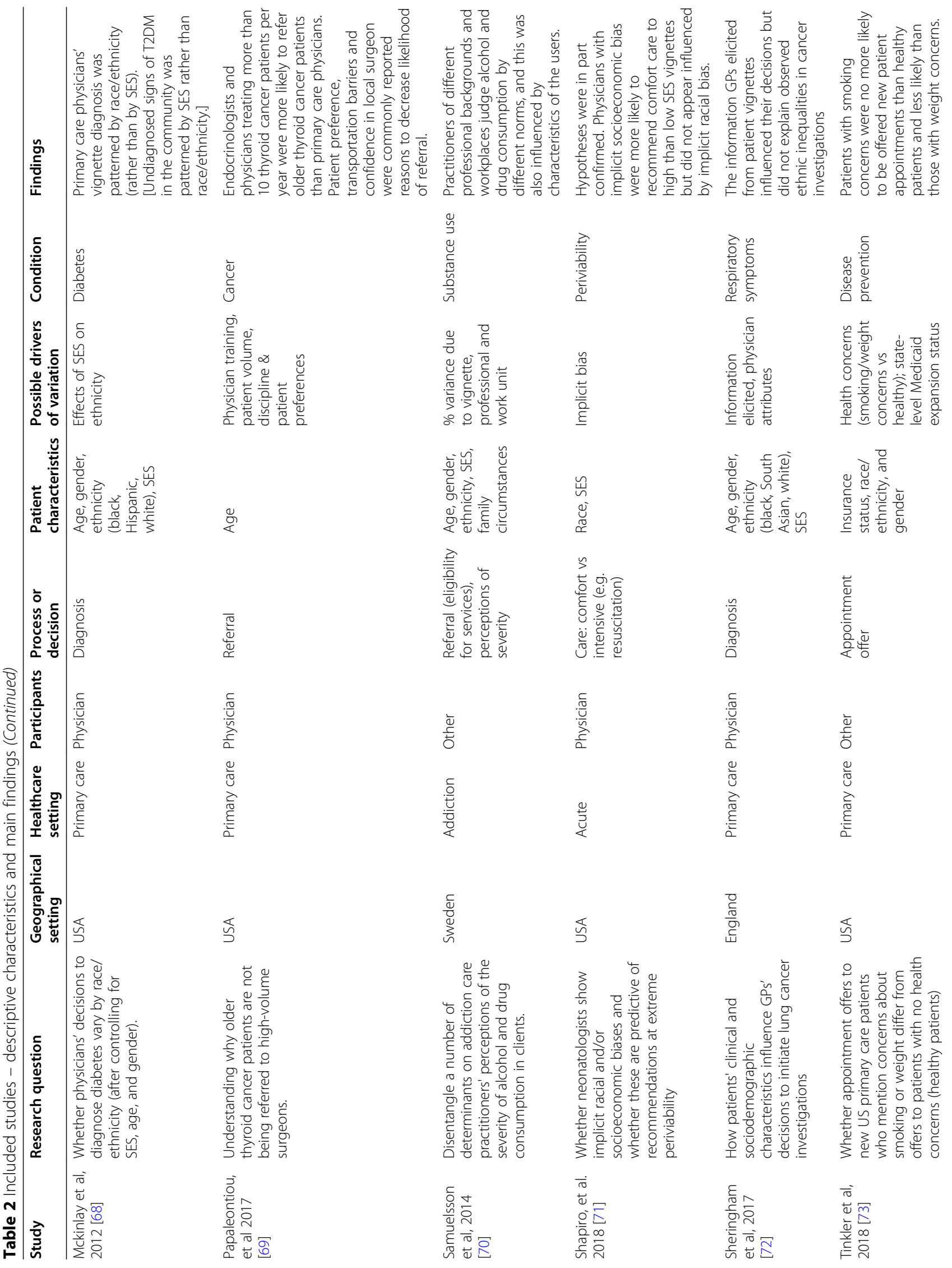




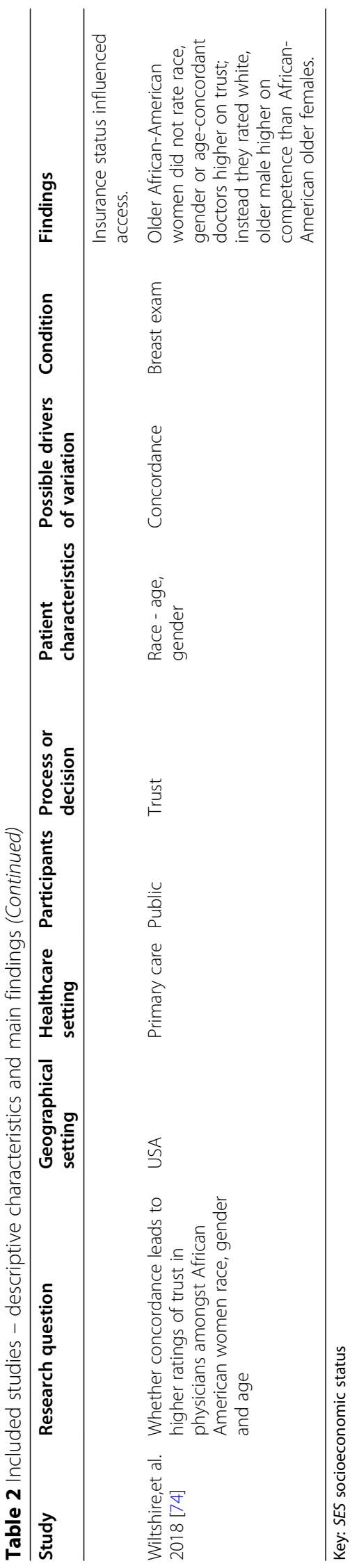




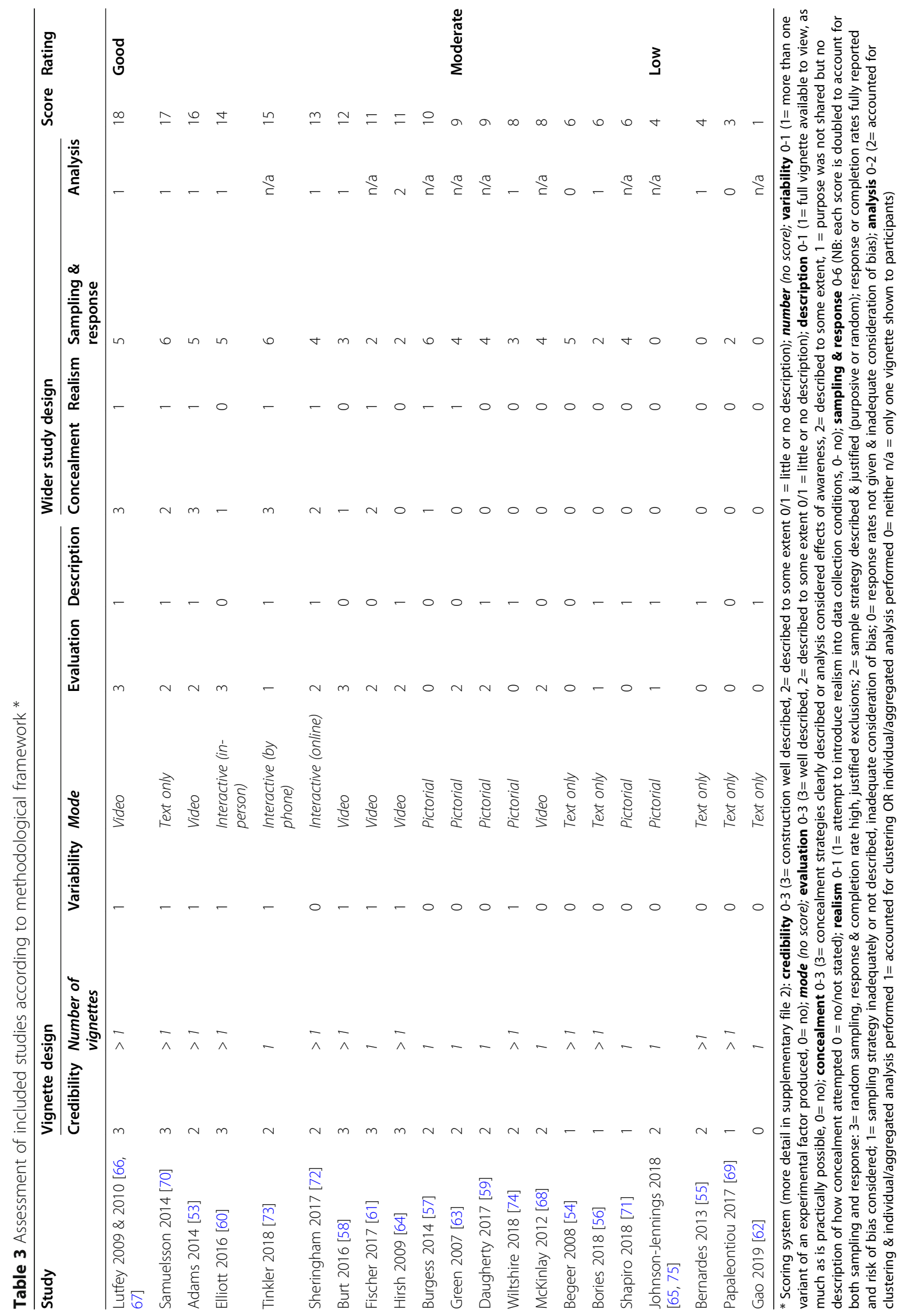




\section{Wider study design \\ Concealment}

While eleven papers reported that the study's purpose was not divulged to participants, only seven high quality described strategies they actively employed to conceal it. These included: stating a wider or different purpose in study information, including a 'distractor' (either an unrelated vignette or unrelated tasks during the study), and using free-text response options as opposed to a predetermined selection $(n=8)$ to reduce the risk of priming and response bias.

Three studies illustrated that participants' awareness of the study purpose could affect the findings [54, 63, 66]. Lutfey et al. (2009) alerted half their sample to the potential of $\mathrm{CHD}$ as a diagnosis; primed doctors made different decisions on the same vignettes to those not explicitly primed [66]. Green et al (2007) found a strong relationship between physicians' implicit bias scores and thrombolysis decisions for black patients in participants unaware of the study's aim; the relationship was reversed in participants aware of the aim [63]. Finally, ethnic bias in the assessment of autism, found when clinicians' were asked to give a spontaneous clinical judgement, disappeared when clinicians were asked to specifically rate the likelihood of autism [54].

Active strategies for concealment were not described in any of the low or moderately rated studies.

\section{Realism}

Six of the moderate and high quality studies sought to collect data in settings that replicated aspects of healthcare delivery, for example by collecting data in physicians' offices during clinic times $[61,66,68]$. Such data collection was not always achieved as planned; Sheringham et al. sought to conduct an online study in clinic settings between appointments, but due to limited clinic IT facilities many participants completed the study at home [72].

\section{Sampling and response rate}

Risk of bias was common due to sampling flaws, low or unreported response rates. It was not limited to low quality studies. Eight studies - two higher, two moderate and all the lower quality studies - lacked explanations or justification of sampling selection, recruitment strategy or representativeness of the final sample. Only eight out of 21 studies reported response rates. Of these, three reported response rates of less than 30\%. Several studies were unable to report the total population contacted for the study due to the method used to approach participants, such as distribution via clinical networks. Insights were also on occasion limited due to challenges of recruitment. Johnson-Jennings et al. sought to examine the extent to which ethnic concordance between clinician and patient was a driver of variations, but insights were limited as they were only able to recruit 33 Native American physicians [75].

\section{Analysis}

Where studies presented respondents with more than one vignette, most sought to control for potential effects of a particular depiction by including the vignette as a covariate in multivariable analysis. Appropriate analytical methods were used to account for clustering. Only two studies sought to examine variation between participants: Bories et al. used clustering to identify characteristics of physician behaviour patterns across vignettes, whilst Hirsh et al. analysed decisions at the level of the individual [56, 64]. This individual-level analysis showed that only a minority of nurses displayed non-clinical variations in decisions, but such variations were sufficiently large to influence the aggregate analyses.

\section{New insights from vignette studies into the drivers of healthcare variations}

Studies contributed to understanding variations in care in two ways. Firstly, most of the moderate or high-quality vignette studies (14/17) sought to test specific hypotheses which might explain observed disparities in care - hypotheses which are challenging to examine using real patients. Secondly, studies aimed to provide insights into poorly understood decision-making processes underlying disparities in care. Many papers served both purposes (testing specific hypotheses and providing new insights), with just three focussing only on insights into decision-making $[53,70,72]$.

Vignette studies may both lend support to, or challenge, hypotheses for how inequalities in healthcare arise. For example, clinicians frequently make decisions amongst competing demands in chaotic working conditions, which result in a background of high cognitive load, and the potential for subsequent variations in care. It is clear that research assessments of decision-making recorded in quiet environments without time constraints do not replicate this pressure. One study provided evidence that bias is more likely to arise in high pressure situations: increasing cognitive load through the provision of a competing task to do under time pressure altered ethnic inequalities in physicians' prescribing patterns [57]. This supports not only the notion that cognitive load leads to variations in care, but that such variations may be systematically biased against certain patient characteristics. Of note, additional cognitive load altered inequalities in prescribing in different ways for male and female physician, highlighting the complexity of contextual influences on disparities in care [57]. 
Two papers used hypotheses generated from real patient data as the basis for tests with parallel vignette studies [54, 68]. Combining insights from observational and vignette data may be particularly helpful in clarifying the relevance of research findings to policy or practice. As an example, in an initial descriptive analysis of case records, Begeer et al. identified that minority ethnic groups were under-represented in autism institutions [54]. In a contemporaneous vignettes study, they found that physicians' ethnic biases in diagnosing autism disappeared when they were specifically prompted to consider autism. The authors suggest the use of structured prompts in clinical assessments may decrease variations in diagnosis and subsequent care [54].

Whilst such insights lend credibility to prior hypotheses, vignette studies may also bring insights that challenge proposed drivers of reported variations in healthcare. For example, Burt et al. noted that certain minority ethnic groups report lower patient experience scores compared to the majority population across a wide variety of settings [58]. One proposed explanation for this is that minority ethnic patients receive similar care to the majority white patients, but have higher or different expectations of care. To test this hypothesis, Burt et al. presented respondents with video vignettes of GP-patient consultations to gauge their expectations of care. They found South Asian respondents consistently rated GPs' communication skills higher than white respondents, thus challenging the hypothesis that poorer reported experiences of care in South Asian patients relative to White British patients arise from higher expectations of care [58].

As noted above, vignette approaches may provide insights into decision-making processes. Three studies in this review sought to obtain new insights into how ethnic disparities arise during healthcare encounters. Obtaining generalisable evidence on this is rarely feasible in real life due to the specific dynamics of individual clinician-patient pairs. Adams et al. asked physicians to reflect on video consultations about depression, analysing these narratives in detail to identify micro-components of clinical decision making [53]. This approach, which yielded rich data on the cues physicians reported using and the inferences they drew from them, in fact suggested there was little ethnic bias in physicians' decision-making processes. Such findings, however, rely on the accuracy of physicians' retrospectively constructed narratives. More recently, two studies used elements of simulation to explore in real time how interactions between patients and healthcare professionals may lead to variations in care [60, 72]. For example, Elliot et al. coded video recordings of encounters between physicians and standardised patients, and demonstrated that variations in healthcare arose during consultations through differences in non-verbal interactions [60].
Studies within this review were able to test hypotheses and generate new insights into decision-making processes through their deliberate divergence from real life situations, involving the manipulation of vignette characteristics and the contexts in which data were collected. As highlighted by many of the studies above, vignette approaches have been particularly useful to date in examining ethnic disparities in care, with researchers circumventing the obstacles experienced in real life of finding sufficient numbers of patients or clinicians of rare ethnicities to undertake studies in this area. The vignette approach also enables standardisation and isolation of characteristics of interest. Such standardisation helps to eliminate the possibility that observed ethnic variations in healthcare delivery were caused by individuals' cultural and linguistic, rather than ethnic group, differences.

\section{Discussion \\ Main findings}

Experimental vignette studies have been used in a number of innovative ways to examine drivers of unwarranted variations in healthcare delivery. They can test hypotheses proposed to explain variations in care that are not possible using real-life data through the manipulation of vignette characteristics or the context in which data were collected.

By applying a novel methodological framework for conducting vignette studies to this review, we demonstrated that their insights have been limited in many cases by a lack of evaluation of the credibility of vignettes and flaws in their wider study design.

\section{Strengths and limitations}

The volume of literature retrieved from the search for empirical studies was large, and in many cases obviously not relevant to the study question. To manage this volume, we instigated an automated screening process, and used limited double screening. As a result, we may not have captured an exhaustive set of all experimental vignette studies identifying drivers of unwarranted variation in healthcare quality. However, our methods were sufficient for our purposes, which were to identify a set of studies of sufficient quality to illustrate the range of ways in which vignette designs have been used and identify areas in which the potential of vignette methods could be maximised to provide further insights into drivers of unwarranted variations in health care.

There were flaws in almost all of the studies retrieved by our search. In most studies, aggregate analyses of decision making were presented, which may mask heterogeneity between physicians' (or patients') perceptions or decision-making behaviour. A number of studies reported findings that were unexpected or counter to 
findings from observational studies. Without searching discussion about why unexpected findings occurred, such vignette studies may have poor credibility and limited capacity to influence future research or policy. More broadly, studies often had severe limitations in their wider design, notably due to biased or incompletely described samples.

\section{What this study adds}

The application of a novel methodological framework to appraise vignette studies illustrated the variation in quality and conduct of such studies. The framework also adds to existing methodological reviews by consolidating guidance into one source and considering the range of modalities - beyond text and video that can be used to depict vignette content [42, 43, 45]. This is important because choice of vignette delivery mode determines what research questions it is possible to answer. For example, if a study seeks to examine events during a clinical encounter, static vignette modalities will not capture these [43]. By illustrating the heterogeneity in reporting in this field, it provides evidence of the need for standard reporting guidelines reflecting the full range of possible vignette modalities to enhance the transparency and quality of vignette studies in health services research.

While developed for appraising studies examining drivers of inequalities, it may have wider applicability to assess the methodological rigour of other experimental vignette studies. This is because most dimensions of the framework - namely considerations of vignette credibility, evaluation and description and the wider study design - are central to vignette studies with any purpose. One dimension - the need to conceal the study purpose - may be more specific to inequalities or to studies seeking to examine behaviours or views that participants feel are undesirable. However, we caution against uncritical application of the scoring system developed for this paper. The scores were weighted to reflect the importance of dimensions considered important in this discipline and may well require adaptation for other fields.

\section{Conclusions}

Understanding how unwarranted variations in healthcare arise is challenging. Experimental vignette studies can help with this, but they need careful design and effort to be conducted to a high standard. To date, most experimental vignette studies have concerned themselves with exploring the attitudes and behaviour of healthcare professionals. There is scope for a greater focus on patient attitudes, experiences and behaviours, and the interactions between patients and providers, in determining how variations arise and persist.

The framework developed in this paper to appraise vignette studies covers dimensions of relevance beyond inequalities. Wider application and adaptation is required to determine the extent to which it can ultimately benefit researchers across scientific disciplines.

\section{Supplementary Information}

The online version contains supplementary material available at https://doi. org/10.1186/s12874-021-01247-4.

\section{Additional file 1}

\section{Acknowledgements}

The authors would like to thank Drs Christian von Wagner, Angelos Kassianos and Ruth Plackett for their constructive comments on an earlier version of this manuscript.

\section{Authors' contributions}

$J B$ and JS had the idea for this study. IK conducted the literature search with all authors devising the search strategy, JB led on construction of the methodological framework; JS and JB screened and extracted data on selected papers. Both JB and JS drafted the manuscript. All authors commented on drafts of the manuscript and agreed the decision to submit for publication. The author(s) read and approved the final manuscript.

\section{Funding}

JS is supported by the National Institute for Health Research Applied Research Collaboration (ARC) North Thames. The views expressed in this publication are those of the authors and not necessarily those of the National Institute for Health Research or the Department of Health and Social Care.

JB and IK are based in The Healthcare Improvement Studies Institute (THIS Institute), University of Cambridge. THIS Institute is supported by the Health Foundation, an independent charity committed to bringing about better health and healthcare for people in the UK.

The funders had no role in study design, data collection and analysis, decision to.

publish, or preparation of the manuscript.

Availability of data and materials

All the data come from peer-reviewed papers listed in the reference list.

\section{Declarations}

Ethics approval and consent to participate Not applicable.

Consent for publication

Not applicable.

Competing interests

The authors declare that they have no competing interests.

Author details

'Department of Applied Health Research, UCL, 1-19 Torrington Place, London WC1E 7HB, UK. ${ }^{2}$ The Healthcare Improvement Studies (THIS) Institute, University of Cambridge, Cambridge Biomedical Campus, Clifford Allbutt Building, Cambridge CB2 OAH, UK. 
Received: 14 November 2020 Accepted: 23 February 2021 Published online: 22 April 2021

\section{References}

1. National Health Service (NHS). The NHS atlas of variation in healthcare series: reducing unwarranted variation to increase value and improve quality. 2016. https://www.england.nhs.uk/rightcare/. Accessed 30 Jan 2021.

2. Dartmouth Atlas of Health Care. Dartm. Atlas Health Care. https://www.da rtmouthatlas.org/. Accessed 30 Jan 2021.

3. Kapilashrami A, Hankivsky O. Intersectionality and why it matters to global health. Lancet. 2018;391:2589-91. https://doi.org/10.1016/S0140-6736(1 8)31431-4.

4. Goldstein E, Elliott MN, Lehrman WG, et al. Racial/ethnic differences in patients' perceptions of inpatient care using the HCAHPS survey. Med Care Res Rev MCRR. 2010;67:74-92. https://doi.org/10.1177/1077558709341066.

5. Weech-Maldonado R, Fongwa MN, Gutierrez $P$, et al. Language and regional differences in evaluations of Medicare managed care by Hispanics. Health Serv Res. 2008;43:552-68. https://doi.org/10.1111/j.1475-6773.2007.00796.x.

6. Lyratzopoulos G, Elliott M, Barbiere JM, et al. Understanding ethnic and other socio-demographic differences in patient experience of primary care: evidence from the English General Practice Patient Survey. BMJ Qual Saf. 2012;21:21-9. https://doi.org/10.1136/bmjqs-2011-000088.

7. Burt J, Lloyd C, Campbell J, et al. Variations in GP-patient communication by ethnicity, age, and gender: evidence from a national primary care patient survey. Br J Gen Pr. 2016;66:e47-52.

8. Lyratzopoulos G, Neal RD, Barbiere JM, et al. Variation in number of general practitioner consultations before hospital referral for cancer: findings from the 2010 National Cancer Patient Experience Survey in England. Lancet Oncol. 2012;13:353-65. https://doi.org/10.1016/S1470-2045(12)70041-4.

9. Zhou Y, Mendonca SC, Abel GA, et al. Variation in 'fast-track' referrals for suspected cancer by patient characteristic and cancer diagnosis: evidence from 670000 patients with cancers of 35 different sites. Br J Cancer. 2018, 118:24-31. https://doi.org/10.1038/bjc.2017.381.

10. Wennberg J, Wennberg JE. Time to tackle unwarranted variations in practice. BMJ. 2011;342:687-90.

11. Katz JN. Patient Preferences and Health Disparities. JAMA. 2001;286:1506-9. https://doi.org/10.1001/jama.286.12.1506.

12. Mulley AG. Improving productivity in the NHS. BMJ. 2010;341:c3965. https:// doi.org/10.1136/bmj.c3965

13. Dehon E, Weiss N, Jones J, et al. A Systematic Review of the Impact of Physician Implicit Racial Bias on Clinical Decision Making. Acad Emerg Med Off J Soc Acad Emerg Med. 2017;24:895-904. https://doi.org/10.1111/a cem.13214.

14. Hall WJ, Chapman MV, Lee KM, et al. Implicit Racial/Ethnic Bias Among Health Care Professionals and Its Influence on Health Care Outcomes: A Systematic Review. Am J Public Health. 2015;105:e60-76. https://doi.org/1 0.2105/AJPH.2015.302903

15. Finch J. The vignette technique in survey research. Sociology. 1987;21:10514.

16. Atzmüller C, Steiner PM. Experimental Vignette Studies in Survey Research. Methodology. 2010;6:128-38. https://doi.org/10.1027/1614-2241/a000014.

17. Herskovits MJ. The hypothetical situation: a technique of field research. Southwest J Anthropol. 1950;6:32-40.

18. Rossi PH, Anderson A. The factorial survey approach: An introduction. In: Rossi PH, Nock S, editors. Measuring social judgments: The factorial survey approach. Thousand Oaks: Sage Publications; 1982

19. Rossi PH, Sampson WA, Bose CE, et al. Measuring household social standing. Soc Sci Res. 1974;3:169-90.

20. Alexander CS, Becker HJ. The use of vignettes in survey research. Public Opin Q. 1978;42:93-104.

21. Schoenberg NE, Ravdal H. Using vignettes in awareness and attitudinal research. Int J Soc Res Methodol. 2000;3:63-74. https://doi.org/10.1080/1364 55700294932

22. Aguinis $\mathrm{H}$, Bradley KJ. Best Practice Recommendations for Designing and Implementing Experimental Vignette Methodology Studies. Organ Res Methods. 2014;17:351-71. https://doi.org/10.1177/1094428114547952.

23. Aiman-Smith L, Scullen SE, Barr SH. Conducting Studies of Decision Making in Organizational Contexts: A Tutorial for Policy-Capturing and Other Regression-Based Techniques. Organ Res Methods. 2002;5:388-414. https:// doi.org/10.1177/109442802237117.
24. Burstin K, Doughtie EB, Raphaeli A. Contrastive vignette technique: An indirect methodology designed to address reactive social attitude measurement. J Appl Soc Psychol. 1980;10:147-65.

25. Hyman MR, Steiner SD. The Vignette Method in Business Ethics Research: Current Uses, Limitations, and Recommendations. Studies. 1996;20:74-100.

26. Evans SC, Roberts MC, Keeley JW, et al. Vignette methodologies for studying clinicians' decision-making: Validity, utility, and application in ICD-11 field studies. Int J Clin Health Psychol. 2015;15:160-70. https://doi.org/10.1016/j. ijchp.2014.12.001.

27. Ludwick R, Wright ME, Zeller RA, et al. An Improved Methodology for Advancing Nursing Research: Factorial Surveys. Adv Nurs Sci. 2004;27:22438. https://doi.org/10.1097/00012272-200407000-00007.

28. Slack M, Draugalis J. Establishing the internal and external validity of experimental studies. Am J Health Syst Pharm. 2001;58:2173.

29. Shadish WR, Cook TD, Campbell DT. Experimental and quasi-experimental designs for generalized causal inference. Belmont: Wadsworth Cengage Learning; 2002

30. Ganong LH, Coleman M. Multiple segment factorial vignette designs. J Marriage Fam. 2006:68:455-68.

31. Steiner PM, Atzmüller C, Su D. Designing Valid and Reliable Vignette Experiments for Survey Research: A Case Study on the Fair Gender Income Gap. J Methods Meas Soc Sci. 2016;7.

32. Hughes R, Huby M. The application of vignettes in social and nursing research. J Adv Nurs. 2002;37:382-6.

33. Peabody JW, Luck J, Glassman P, et al. Comparison of vignettes, standardized patients, and chart abstraction: A prospective validation study of 3 methods for measuring quality. JAMA. 2000;283:1715-22. https://doi. org/10.1001/jama.283.13.1715.

34. Peabody JW, Luck J, Glassman P, et al. Measuring the Quality of Physician Practice by Using Clinical Vignettes: A Prospective Validation Study. Ann Intern Med. 2004;141:771. https://doi.org/10.7326/0003-4819-141-10-2004111 60-00008.

35. Shah R, Edgar DF, Evans BJW. A comparison of standardised patients, record abstraction and clinical vignettes for the purpose of measuring clinical practice. Ophthalmic Physiol Opt. 2010;30:209-24. https://doi.org/10.1111/ j.1475-1313.2010.00713.x.

36. Amelung D, Whitaker KL, Lennard D, et al. Influence of doctor-patient conversations on behaviours of patients presenting to primary care with new or persistent symptoms: a video observation study. BMJ Qual Saf. 2019: bmjqs-2019-009485. https://doi.org/10.1136/bmjqs-2019-009485.

37. Hrisos S, Eccles MP, Francis JJ, et al. Are there valid proxy measures of clinical behaviour? A systematic review. Implement Sci IS. 2009:4:37. https:// doi.org/10.1186/1748-5908-4-37.

38. Sepucha KR, Abhyankar P, Hoffman AS, et al. Standards for UNiversal reporting of patient Decision Aid Evaluation studies: the development of SUNDAE Checklist. BMJ Qual Saf. 2018;27:380-8. https://doi.org/10.1136/ bmjas-2017-006986.

39. Tricco AC, Lillie E, Zarin W, et al. PRISMA Extension for Scoping Reviews (PRISMA-SCR): Checklist and Explanation. Ann Intern Med. 2018;169:467. https://doi.org/10.7326/M18-0850.

40. Stata Statistical Software: Release 15. College Station: StataCorp LLC. 2017.

41. McHugh ML. Interrater reliability: the kappa statistic. Biochem Medica. 2012; 22:276-82.

42. Veloski J, Tai S, Evans AS, et al. Clinical Vignette-Based Surveys: A Tool for Assessing Physician Practice Variation. Am J Med Qual. 2005;20:151-7. https://doi.org/10.1177/1062860605274520.

43. Bachmann LM, Mühleisen A, Bock $A$, et al. Vignette studies of medical choice and judgement to study caregivers' medical decision behaviour: systematic review. BMC Med Res Methodol. 2008;8:50. https://doi.org/10.11 86/1471-2288-8-50.

44. van Vliet LM, Hillen MA, van der Wall E, et al. How to create and administer scripted video-vignettes in an experimental study on disclosure of a palliative breast cancer diagnosis. Patient Educ Couns. 2013;91:56-64. https://doi.org/10.1016/j.pec.2012.10.017.

45. Hillen MA, van Vliet LM, de Haes HCJM, et al. Developing and administering scripted video vignettes for experimental research of patient-provider communication. Patient Educ Couns. 2013;91:295-309. https://doi.org/10.1 016/j.pec.2013.01.020

46. Converse L, Barrett $K$, Rich E, et al. Methods of Observing Variations in Physicians' Decisions: The Opportunities of Clinical Vignettes. J Gen Intern Med. 2015;30:586-94. https://doi.org/10.1007/s11606-015-3365-8. 
47. Karelaia N, Hogarth RM. Determinants of linear judgment: A meta-analysis of lens model studies. Psychol Bull. 2008;134:404-26. https://doi.org/10.1037/ 0033-2909.134.3.404

48. Hirsch $\mathrm{O}$, Keller $\mathrm{H}$, Albohn-Kuhne $\mathrm{C}$, et al. Pitfalls in the statistical examination and interpretation of the correspondence between physician and patient satisfaction ratings and their relevance for shared decision making research. BMC Med Res Methodol. 2011;11:71. https://doi.org/10.11 86/1471-2288-11-71.

49. Wallander L. 25 years of factorial surveys in sociology: A review. Soc Sci Res. 2009;38:505-20. https://doi.org/10.1016/j.ssresearch.2009.03.004.

50. Eccles MP, Grimshaw JM, Johnston M, et al. Applying psychological theories to evidence-based clinical practice: Identifying factors predictive of managing upper respiratory tract infections without antibiotics. Implement Sci. 2007;2:26. https://doi.org/10.1186/1748-5908-2-26.

51. Kelley K, Clark B, Brown V, et al. Good practice in the conduct and reporting of survey research. Int J Qual Health Care. 2003;15:261-6. https://doi.org/1 0.1093/intqhc/mzg031

52. Eccles MP, Grimshaw JM, MacLennan G, et al. Explaining clinical behaviors using multiple theoretical models. Implement Sci. 2012;7:99. https://doi. org/10.1186/1748-5908-7-99.

53. Adams A, Vail L, Buckingham CD, et al. Investigating the influence of African American and African Caribbean race on primary care doctors' decision making about depression. Soc Sci Med. 2014;116:161-8. https://doi.org/10.1 016/j.socscimed.2014.07.004.

54. Begeer S, Bouk SE, Boussaid W, et al. Underdiagnosis and Referral Bias of Autism in Ethnic Minorities. J Autism Dev Disord. 2008;39:142. https://doi. org/10.1007/s10803-008-0611-5.

55. Bernardes SF, Costa M, Carvalho H. Engendering Pain Management Practices: The Role of Physician Sex on Chronic Low-Back Pain Assessment and Treatment Prescriptions. J Pain. 2013;14:931-40. https://doi.org/10.1016/ j.jpain.2013.03.004

56. Bories $\mathrm{P}$, Lamy $\mathrm{S}$, Simand $\mathrm{C}$, et al. Physician uncertainty aversion impacts medical decision making for older patients with acute myeloid leukemia: results of a national survey. Haematologica. 2018;103:2040-8. https://doi. org/10.3324/haematol.2018.192468.

57. Burgess DJ, Phelan S, Workman M, et al. The effect of cognitive load and patient race on physicians' decisions to prescribe opioids for chronic low back pain: a randomized trial. Pain Med. 2014;15:965-74.

58. Burt J, Abel G, Elmore N, et al. Understanding negative feedback from South Asian patients: an experimental vignette study. BMJ Open. 2016;6:e011256.

59. Daugherty SL, Blair IV, Havranek EP, et al. Implicit Gender Bias and the Use of Cardiovascular Tests Among Cardiologists. J Am Heart Assoc. 2017;6. https://doi.org/10.1161/JAHA.117.006872.

60. Elliott AM, Alexander SC, Mescher CA, et al. Differences in Physicians' Verbal and Nonverbal Communication With Black and White Patients at the End of Life. J Pain Symptom Manag. 2016;51:1-8. https://doi.org/10.1016/j.jpa insymman.2015.07.008.

61. Fischer MA, McKinlay JB, Katz JN, et al. Physician assessments of drug seeking behavior: A mixed methods study. PLoS One. 2017;12:e0178690.

62. Gao S, Corrigan PW, Qin S, et al. Comparing Chinese and European American mental health decision making. J Ment Health. 2019;28:141-7. https://doi.org/10.1080/09638237.2017.1417543.

63. Green AR, Carney DR, Pallin DJ, et al. Implicit Bias among Physicians and its Prediction of Thrombolysis Decisions for Black and White Patients. J Gen Intern Med. 2007;22:1231-8. https://doi.org/10.1007/s11606-007-0258-5.

64. Hirsh AT, George SZ, Robinson ME. Pain Assessment and Treatment Disparities: A Virtual Human Technology Investigation. Pain. 2009;143:10613. https://doi.org/10.1016/j.pain.2009.02.005.

65. Johnson-Jennings M, Walters K, Little M. And [They] Even Followed Her Into the Hospital: Primary Care Providers' Attitudes Toward Referral for Traditional Healing Practices and Integrating Care for Indigenous Patients. J Transcult Nurs Off J Transcult Nurs Soc. 2018;29:354-62. https://doi.org/10.11 77/1043659617731817

66. Lutfey KE, Link CL, Marceau LD, et al. Diagnostic Certainty as a Source of Medical Practice Variation in Coronary Heart Disease: Results from a CrossNational Experiment of Clinical Decision Making. Med Decis Mak. 2009;29: 606-18. https://doi.org/10.1177/0272989X09331811.

67. Lutfey KE, Eva KW, Gerstenberger E, et al. Physician cognitive processing as a source of diagnostic and treatment disparities in coronary heart disease: results of a factorial priming experiment. J Health Soc Behav. 2010;51:16-29.
68. McKinlay JB, Marceau LD, Piccolo RJ. Do doctors contribute to the social patterning of disease: the case of race/ethnic disparities in diabetes mellitus. Med Care Res Rev. 2012;69:176-93.

69. Papaleontiou M, Gauger PG, Haymart MR. Referral of older thyroid cancer patients to a high-volume surgeon: Results of a multidisciplinary physician survey. Endocr Pract Off J Am Coll Endocrinol Am Assoc Clin Endocrinol. 2017;23:808-15. https://doi.org/10.4158/EP171788.OR.

70. Samuelsson E, Wallander L. Disentangling practitioners' perceptions of substance use severity: A factorial survey. Addict Res Theory. 2014;22:34860. https://doi.org/10.3109/16066359.2013.856887.

71. Shapiro N, Wachtel EV, Bailey SM, et al. Implicit Physician Biases in Periviability Counseling. J Pediatr. 2018;197:109-115.e1. https://doi.org/10.1 016/j.jpeds.2018.01.070

72. Sheringham J, Sequeira R, Myles J, et al. Variations in GPs' decisions to investigate suspected lung cancer: a factorial experiment using multimedia vignettes. BMJ Qual Saf. 2017;26:449-59. https://doi.org/10.1136/bmjqs-201 6-005679.

73. Tinkler SE, Sharma RL, Susu-Mago RRH, et al. Access to US primary care physicians for new patients concerned about smoking or weight. Prev Med. 2018;113:51-6. https://doi.org/10.1016/j.ypmed.2018.04.031.

74. Wiltshire J, Allison JJ, Brown R, et al. African American women perceptions of physician trustworthiness: A factorial survey analysis of physician race, gender and age. AIMS Public Health. 2018;5:122-34. https://doi.org/10.3934/ publichealth.2018.2.122.

75. Johnson-Jennings M, Tarraf W, González HM. The healing relationship in Indigenous patients' pain care: Influences of racial concordance and patient ethnic salience on healthcare providers' pain assessment. Int J Indig Health. 2015;10:33.

76. Blair IV, Steiner JF, Fairclough DL, et al. Clinicians' Implicit Ethnic/Racial Bias and Perceptions of Care Among Black and Latino Patients. Ann Fam Med. 2013;11:43-52. https://doi.org/10.1370/Afm.1442.

\section{Publisher's Note}

Springer Nature remains neutral with regard to jurisdictional claims in published maps and institutional affiliations.

Ready to submit your research? Choose BMC and benefit from

- fast, convenient online submission

- thorough peer review by experienced researchers in your field

- rapid publication on acceptance

- support for research data, including large and complex data types

- gold Open Access which fosters wider collaboration and increased citations

- maximum visibility for your research: over $100 \mathrm{M}$ website views per year

At $\mathrm{BMC}$, research is always in progress.

Learn more biomedcentral.com/submissions 